\title{
Cerebrospinal fluid total protein determination in acute and chronic inflammatory demyelinating polyneuropathies: a critical reappraisal
}

\section{Dear Editor,}

Albumino-cytologic dissociation is a typical feature of Guillain-Barré syndrome (GBS) and its variants (Fokke et al., 2014), and of chronic inflammatory demyelinating polyradiculoneuropathy (CIDP), representing one of the supportive criteria for the diagnosis (Joint Task Force of the EFNS and the $P N S$, 2010). Notwithstanding the explicit reference to the term "albumin", the criteria entail that the dissociation is evaluated by measuring cerebrospinal fluid (CSF) total proteins and counting CSF cells. Semantic violation apart, the topic deserves a reappraisal, since using CSF total proteins as a marker of inflammation-dependent increased permeability of the blood-CSF barrier (B-CSF-B) might lead to inaccurate evaluations. The calculation of the most accurate albumin quotient $\left(\mathrm{Q}_{\text {Alb }}=\mathrm{CSF}\right.$ albumin/serum albumin) has long substituted for CSF total proteins in the area of multiple sclerosis and other acquired demyelinating syndromes (Andersson et al., 1994; Gastaldi et al., 2017).

Preliminarily, it is worth noting that inflammation in GBS/CIDP affects the blood-spinal nerve root barrier (B-SNR-B), but not the B-CSF-B, formed by choroid plexuses, nor the blood-brain barrier, whose increased permeability, in turn, cannot be evaluated with the CSF analysis (Thompson, 1988), as not infrequently done (e.g., Stich et al., 2016).

Another important physiological aspect is that $\sim 80 \%$ of the CSF proteins are plasma-derived and only $\sim 20 \%$ intrathecally produced (Thompson, 1988). Therefore, assessing the degree of B-SNR-B/B-CSF-B permeability as an index of spinal nerve roots/intrathecal inflammation by means of CSF total protein concentrations implies two shortcomings: (1) serum total protein concentrations, on which CSF total protein concentrations strictly depend,

Address correspondence to: Diego Franciotta, IRCCS Mondino Foundation, via Mondino 2, I-27100 Pavia, Italy. Tel: +(39)0382-380365; Fax: +(39)0382-380286; E-mail: diego.franciotta@mondino.it are not considered and (2) intrathecally produced proteins - mainly immunoglobulin G (lgG) during immuno-inflammatory CNS disorders - can be considered as part of the bulk of CSF total proteins although their CSF concentrations are obviously independent from any increase of B-SNR-B/B-CSF-B permeability.

As a result, patients with chronic or transient low concentrations of plasma proteins (e.g., from kidney or liver diseases) might show "normal" CSF total protein concentrations even in the presence of a leaky B-SNR-B/B-CSF-B (false negative results); vice versa, patients with abnormally high concentrations of plasma proteins (e.g., paraproteinemic patients; patients with previous therapy with intravenous immunoglobulins), or with intrathecal $\lg G$ production (e.g., from chronic immuno-inflammatory CNS disorders or combined central and peripheral demyelination) might have "abnormal" CSF total protein concentrations notwithstanding a preserved B-SNR-B/B-CSF-B permeability (false positive results).

Albumin is produced by liver only, is not catabolized in the CNS, and thus, originating exclusively from blood, represents an ideal molecule to assess B-SNR-B/B-CSF-B permeability, as long as its serum concentrations are compared with the CSF concentrations (Thompson, 1988; Andersson et al., 1994). It can be immuno-nephelometrically/-turbidimetrically measured in serum and CSF with automated analyzers and the ensuing $\mathrm{Q}_{\mathrm{Alb}}$, being a pure number, has also the analytical advantage of compensating for inaccuracies in measurements and for biological variability (Thompson, 1988; Infusino and Panteghini, 2013). Moreover, colorimetry, commonly used for CSF total protein determinations, is less accurate than immuno-nephelometry/-turbidimetry (Infusino and Panteghini, 2013).

As a proof of principle, to address the topic we measured total protein concentrations in 50 properly stored (Teunissen et al., 2009) CSF samples (turbidimetry, Cobas Core, Roche Diagnostics) from 
Table 1. Examples of false negative values of cerebrospinal fluid total protein concentrations as compared with the albumin quotient values.

\begin{tabular}{lcccc}
\hline Patient & Diagnosis & $\begin{array}{c}\text { Topic-related } \\
\text { laboratory characteristic }\end{array}$ & $\begin{array}{c}\text { Albumin quotient* } \\
(<0.70)\end{array}$ & $\begin{array}{c}\text { CSF total protein } \\
(<45.0 \mathrm{mg} / \mathrm{dl})\end{array}$ \\
\hline 1 & Optic neuritis & None & 0.80 & 44.0 \\
2 & Mild cognitive impairment & Hypoalbuminemia, 2,515 mg/dl & 1.00 & 42.1 \\
3 & Primary cerebral vasculitis & Hypoalbuminemia, 2,291 mg/dl & 0.79 & 29.2 \\
\hline
\end{tabular}

Reference values are indicated in parentheses.

CSF, cerebrospinal fluid.

${ }^{*}$ Calculated as CSF albumin/serum albumin \%.

neurological patients who underwent lumbar puncture for diagnostic purposes. CSF total protein concentrations correlated with the respective $\mathrm{Q}_{\text {Alb }}$ values that had been determined as part of our CSF routine diagnostics $\left(R^{2}=0.93 ; p=0.002\right)$. However, in three cases (two of them characterized by hypoalbuminemia) $\mathrm{Q}_{\text {Alb }}$ values were abnormally increased, whereas CSF total protein concentration were within the reference range (Table 1), while in another three patients with high values of intrathecal IgG production, CSF total protein concentrations were near the upper reference range, whereas $\mathrm{Q}_{\text {Alb }}$ values well within the reference range (not shown). These findings show that especially false negative results can occur in establishing the inflammation-related increases in B-SNR-B/B-CSF-B permeability, if evaluations of CSF total protein concentrations are used. Considering a routine analysis on unselected CSF samples, our findings suggest that the misclassification rate might be low, but not negligible $(\sim 5 \%)$, affecting cases with values near the reference ranges. Noteworthily, neuroimmunology laboratories currently measure serum and CSF albumin for assessing intrathecal IgG production (IgG index and Reiber's $\lg G_{\text {Loc }}$ ), and therefore $Q_{\text {Alb }}$ could be calculated without extra costs.

Both CSF total protein concentrations and $\mathrm{Q}_{\text {Alb }}$ values are in inverse correlation with the CSF flow rate, which physiologically decreases with age. Their respective age-related upper reference limits have been recently assessed on a large number of patients and useful formulae were produced for the age-related corrections (Hegen et al., 2016). Again, the limits of total proteins in the CSF were reported without considering their dependence on serum concentrations.

In summary, although CSF total protein determinations could fulfill the diagnostic needs in the majority of GBS/CIDP cases, the easy-to-obtain and more accurate $\mathrm{Q}_{\text {Alb }}$ should be considered at least in patients with diseases affecting total serum protein concentrations and in those with diseases associated with intrathecal IgG production. $\mathrm{Q}_{\text {Alb }}$ could also improve accuracy in the evaluation of B-SNR-B/B-CSF-B permeability in multicenter research settings.

\section{Disclosure}

No potential conflict of interest is reported by the authors.

\section{Acknowledgement}

This work was supported by the Italian Ministry of Health 'Ricerca Corrente' 2017-2019 Grant to IRCCS Mondino Foundation.

Sincerely, Diego Franciotta ${ }^{1}$, Matteo Gastaldi ${ }^{1,2}$, Elisabetta Zardini ${ }^{1,2}$, and Eduardo Nobile-Orazio ${ }^{3}$

${ }^{1}$ Laboratory of Neuroimmunology, IRCCS Mondino Foundation, Pavia, Italy; ${ }^{2}$ Department of Brain and Behavioral Sciences, University of Pavia, Pavia, Italy; and ${ }^{3}$ Neuromuscular and Neuroimmunology Service, Department of Medical Biotechnology and Translational Medicine, Humanitas Clinical and Research Institute, Milan University, Milan, Italy

\section{References}

Andersson M, Alvarez-Cermeño J, Bernardi G, Cogato I, Fredman P, Frederiksen J, Fredrikson S, Gallo P, Grimaldi LM, Grønning M, Keir G, Lamers K, Link H, Magalhaes A, Massaro AR, Ohman S, Reiber H, Rönnback L, Schluep M, Schuller E, Sindic CJM, Thompson EJ, Trojano M, Wurster $U$ (1994). Cerebrospinal fluid in the diagnosis of multiple sclerosis: a consensus report. J Neurol Neurosurg Psychiatry 57:897-902.

Fokke C, van den Berg B, Drenthen J, Walgaard C, van Doorn PA, Jacobs BC (2014). Diagnosis of Guillain-Barré syndrome and validation of Brighton criteria. Brain 137:33-43.

Gastaldi M, Zardini E, Franciotta D (2017). An update on the use of cerebrospinal fluid analysis as a diagnostic tool in multiple sclerosis. Expert Rev Mol Diagn 17:31-46.

Hegen H, Auer M, Zeileis A, Deisenhammer F (2016). Upper reference limits for cerebrospinal fluid total protein and albumin quotient based on a large cohort of control patients: implications for increased clinical specificity. Clin Chem Lab Med 54:285-292.

Infusino I, Panteghini M (2013). Serum albumin: accuracy and clinical use. Clin Chim Acta 419:15-18. 
Joint Task Force of the EFNS and the PNS (2010). European Federation of Neurological Societies/Peripheral Nerve Society Guideline on management of chronic inflammatory demyelinating polyradiculoneuropathy: report of a joint task force of the European Federation of Neurological Societies and the Peripheral Nerve Society--first revision. J Peripher Nerv Syst 15:1-9.

Stich O, Glos D, Brendle M, Dersch R, Rauer S (2016). Cerebrospinal fluid profile and seroprevalence of antiganglioside reactivity in patients with neuralgic amyotrophy. J Peripher Nerv Syst 21:27-32.
Teunissen CE, Petzold A, Bennett JL, Berven FS, Brundin L, Comabella M, Franciotta D, Frederiksen JL, Fleming JO, Furlan R, Hintzen RQ, Hughes SG, Johnson MH, Krasulova E, Kuhle J, Magnone MC, Rajda C, Rejdak K, Schmidt HK, van Pesch V, Waubant E, Wolf C, Giovannoni G, Hemmer B, Tumani $H$, Deisenhammer $F$ (2009). A consensus protocol for the standardization of cerebrospinal fluid collection and biobanking. Neurology 73:1914-1922.

Thompson EJ (1988). The CSF Proteins: A Biochemical Approach. Elsevier, Amsterdam, The Netherlands. 